\title{
Intensity stabilisation of optical pulse sequences for coherent control of laser-driven qubits
}

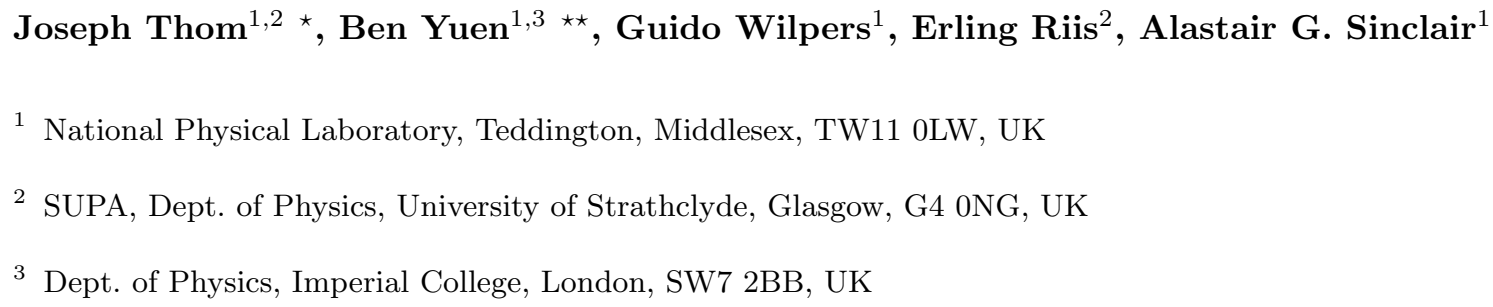

\begin{abstract}
We demonstrate a system for intensity stabilisation of optical pulse sequences used in laser-driven quantum control of trapped ions. Intensity instability is minimised by active stabilisation of the power (over a dynamic range of $>10^{4}$ ) and position of the focused beam at the ion. The fractional Allan deviations in power were found to be $<2.2 \times 10^{-4}$ for averaging times from $1 \mathrm{~s}$ to 16384 s. Over similar times, the absolute Allan deviation of the beam position is $<0.1 \mu \mathrm{m}$ for a $45 \mu \mathrm{m}$ beam diameter. Using these residual power and position instabilities, we estimate the associated contributions to infidelity in example qubit logic gates to be below $10^{-6}$ per gate.
\end{abstract}

* Present address: M Squared Lasers, 1 Kelvin Campus, Glasgow, G20 0SP, UK

$\star \star$ Present address: Dept. of Physics, University of Oxford, Clarendon Laboratory, Parks Road, Oxford, OX1 3PU

\section{Introduction}

Scalable quantum computation requires not only logical qubits, but also many physical qubits and gates for the purpose of error-correction. The number of physical qubits per logical qubit depends strongly on the accuracy of controlling the latter [1]; this number diverges as the error probability per logic gate (EPG) increases to a limiting threshold. Device capabilities, error models and associated assumptions determine the exact threshold for the EPG, which can range from $10^{-2}$ to $10^{-6}[2$, 3]. The consequence of tolerating a high EPG threshold is a requirement for a much larger number of physical qubits. To achieve fault-tolerance without an excessive overhead of physical qubits per logical qubit, the general consensus is that the EPG (or infidelity) must be $<10^{-4}$ $[2,4]$. 
For two decades, much research has been conducted into the use of trapped atomic ions for quantum information processing [5]. A number of landmark ion trap experiments have demonstrated laser-driven two-qubit gates, with infidelities reducing by over two orders of magnitude during this period [6-10]. Trapped ions are at the forefront of high-fidelity quantum gates $[4,11]$, where one of the present challenges is routine achievement of the EPG below a $10^{-4}$ threshold $[12,13]$. Therefore, all effects which contribute $\sim 10^{-4}$ or more to the overall gate error will need to be minimised further.

In coherent control schemes for trapped ions, several noise sources and imperfections are known to introduce errors to qubit operations. These encompass instabilities in the trapping potential, motional heating of the ions, off-resonant excitations and ac-Stark shifts, state preparation and measurement errors, as well as instabilities in the phase and frequency of the coherent driving field. Depending on the choice of species and qubit, magnetic field fluctuations and spontaneous scattering may be additional limitations. Considering laser-driven qubit transitions, instability in the Rabi frequency which characterises the ion-laser interaction [14] will likewise contribute to the overall error. Consequently, the instability of laser intensity experienced by the ions is critical to laser-driven qubits; this is true for both optical transitions as well as hyperfine transitions driven by a stimulated Raman process. In the case of single-qubit gates using hyperfine transitions, lower errors have been achieved by driving the transition directly with a microwave source $[15,16]$. Nevertheless, there remains considerable interest in laser-driven two-qubit gates based on such transitions. The work described here focuses exclusively on methods to minimise intensity instability in optical pulse sequences for coherent control of trapped ions.

Laser intensity fluctuations are considered as a contribution to overall quantum gate error in recent works using hyperfine qubits in ${ }^{9} \mathrm{Be}^{+}[4]$ and ${ }^{43} \mathrm{Ca}^{+}[11]$. Both works report laser power stabilisation as part of the apparatus, with the latter achieving $\sim 10^{-3}$ power instability, which contributed $1 \times 10^{-4}$ to the gate error budget. Similar considerations are made in the report of a twoelement logic gate of ${ }^{9} \mathrm{Be}^{+}$and ${ }^{25} \mathrm{Mg}^{+}[17]$, as well as in earlier work with ${ }^{9} \mathrm{Be}^{+}[18,19]$. In work with ${ }^{40} \mathrm{Ca}^{+}$optical qubits [10], average laser intensity noise of $\sim 4 \times 10^{-3}$ was quantified through Ramsey experiments [20]. Furthermore, active stabilisation of laser intensity has been mentioned in the context of the ${ }^{88} \mathrm{Sr}^{+}$optical qubit transition at $674 \mathrm{~nm}[21,22]$.

In this work we present a laser system with a high degree of intensity agility, while still achieving stability in power and beam pointing over the short and long term ( $\sim 1$ s and $\sim 1$ day respectively). This system was developed for pulsed-probe, coherent control of trapped ions. The parameters of the laser beam are sensed by photodiodes. This approach is preferable to using the response of trapped ions, since it provides for superior 
signal-to-noise. With the pulse power and beam positions stabilised independently, the respective Allan deviations were measured to quantify short- and long-term instabilities. We demonstrate an out-of-loop power instability an order of magnitude lower than reported elsewhere $[4,11]$. The optical method used in this work is not subject to the systematic drifts (e.g. micromotion) which may be present when relying on the ion response as the sole discriminant for quantifying intensity instability. Nevertheless, in the system's intended application to trapped ions, it will still be necessary to initialise the exact intensity set-point via calibration of the Rabi frequency using the ion response. Upon correction of systematic drifts in this response (at intervals dependent on the individual apparatus), the long-term intensity stability will enable restoration of the initial Rabi frequency; otherwise, a recalibration will be required.

Additionally, the system can operate over a range of four orders of magnitude in intensity. Higher intensities enable fast qubit rotations, whereas the lowest intensities are used in Rabi and Ramsey spectroscopy to enable high resolution and measurement accuracy. The system builds on our earlier techniques for agility in pulse shape, phase and frequency detuning [23]. While the principle of the system is demonstrated at a wavelength of $674 \mathrm{~nm}$ for the optical qubit transition in ${ }^{88} \mathrm{Sr}^{+}$, it is straightforwardly applicable to laser-driven transitions in other atomic species.
The experimental data presented here was used to estimate the contribution of laser intensity instability (through Rabi frequency fluctuations) to the infidelity in two examples of laser-driven quantum gates. Our estimates show that this specific source of infidelity can be rendered insignificant with respect to the generallyaccepted EPG threshold of $10^{-4}[2,4]$. It is advantageous to minimise all error sources well below this threshold, since the error of a computation grows rapidly with number of gate operations [10] and the total error determines the magnitude of resources required for error correction $[1]$

\section{Pulse power calibration and stabilisation}

Here we present the principle of a pulse-intensity stabilisation scheme, which is designed for high-fidelity coherent control of the $5 \mathrm{~s}{ }^{2} \mathrm{~S}_{1 / 2}-4 \mathrm{~d}{ }^{2} \mathrm{D}_{5 / 2}$ quadrupole transition at $674 \mathrm{~nm}$ in ${ }^{88} \mathrm{Sr}^{+}$. In such an experiment, using the ion response as a discriminant is problematic at short times due to the quantum projection noise limit, and at long times due to drifts in other parameters which influence the Rabi frequency. Therefore it is essential to employ a method independent of ions, to stabilise the intensity and to quantify the instability achieved. The system described here uses a cascaded series of avalanche photodiodes (APDs) to measure indirectly the power of laser pulses incident on the position of a trapped ion, over a power range of four orders of magnitude. We 
demonstrate long-term stabilisation over the full range of available intensities.

\subsection{Power stabilisation system - experimental setup}

A $674 \mathrm{~nm}$ titanium-sapphire laser (M Squared Lasers SolsTiS) is attenuated to $110 \mathrm{~mW}$. This output is coupled into a polarisation-maintaining (PM) fibre, which delivers the light to a pair of acousto-optic modulators (AOMs) in series (see Fig. 1). AOM1 is used in a doublepass configuration and is driven by the amplified signal from a direct digital synthesis (DDS) source (Toptica Photonics VFG150); this precisely controls the temporal shape, phase, and frequency detuning of the optical pulses, as well as setting the initial power of each pulse over a large dynamic range. An analogue signal generator, in line with a variable attenuator (Minicircuits ZX73-2500-S+) and an amplifier, drives AOM2 in single pass configuration. This permits fine control of the applied RF power, thus enabling precise laser power stabilisation. In the 'off' state, each AOM has the RF extinguished by an $80 \mathrm{~dB}$ switch (Minicircuits ZASWA$2-50 \mathrm{DR}+)$.

A second PM fibre delivers light to the ion, as well as to a series of detectors for optical response calibration and pulse power stabilisation (see Fig. 1). Light from the fibre is collimated by a multi-element achromatic lens (L1, $f=29 \mathrm{~mm}$ ); then, a Glan-Taylor polariser produces a vertical linear polarisation of high purity $\left(10^{5}: 1\right.$ extinction ratio). An achromatic doublet lens
(L2, $f=200 \mathrm{~mm}$ ) focuses the beam to a spot diameter $2 w_{0}=45 \mu \mathrm{m}$ at the position of the ion. This beam is split into two separate paths using a non-polarising 50:50 beamsplitter (Thorlabs BSW10). While the reflected beam is directed to the ion trap, the transmitted beam (of power $P_{\text {in-loop }}$ ) is directed towards a cascaded arrangement of APDs (Hamamatsu C10508-01). Samples of signals from APD1, APD2 or APD3 during optical pulses are used to generate a stabilising feedback signal; these APDs are referred to as the 'in-loop' detectors. APD1 measures the transmitted beam through the wedged optic (0.942 $\left.P_{\text {in-loop }}\right)$, APD2 measures the first reflection $\left(3.79 \times 10^{-2} P_{\text {in-loop }}\right)$ and APD3 measures the second reflection $\left(1.25 \times 10^{-3} P_{\text {in-loop }}\right)$.

When used for coherent control of trapped ions, the reflection from the beamsplitter (power $P$ ) is directed through the ion trap and onward to the position stabilisation system. For the purposes of measuring the efficacy of the power stabilisation system, this beam is directed to a second detection setup to provide an independent, 'out-of-loop' measurement of the pulse powers. In the case where samples of APD1, APD2, or APD3 are used to generate feedback, APD0 (also Hamamatsu C1050801) monitors the beam in position $\mathrm{A}, \mathrm{B}$ or $\mathrm{C}$ respectively. As the setup is similar to that used for the in-loop detectors, the optical powers incident on the in- and out-ofloop detectors are approximately equal. Therefore, for a pulse of a given power $P$ at the ion in the range $300 \mathrm{nW} \leq P \leq 5.2 \mathrm{~mW}$, at least one of APD1, APD2 


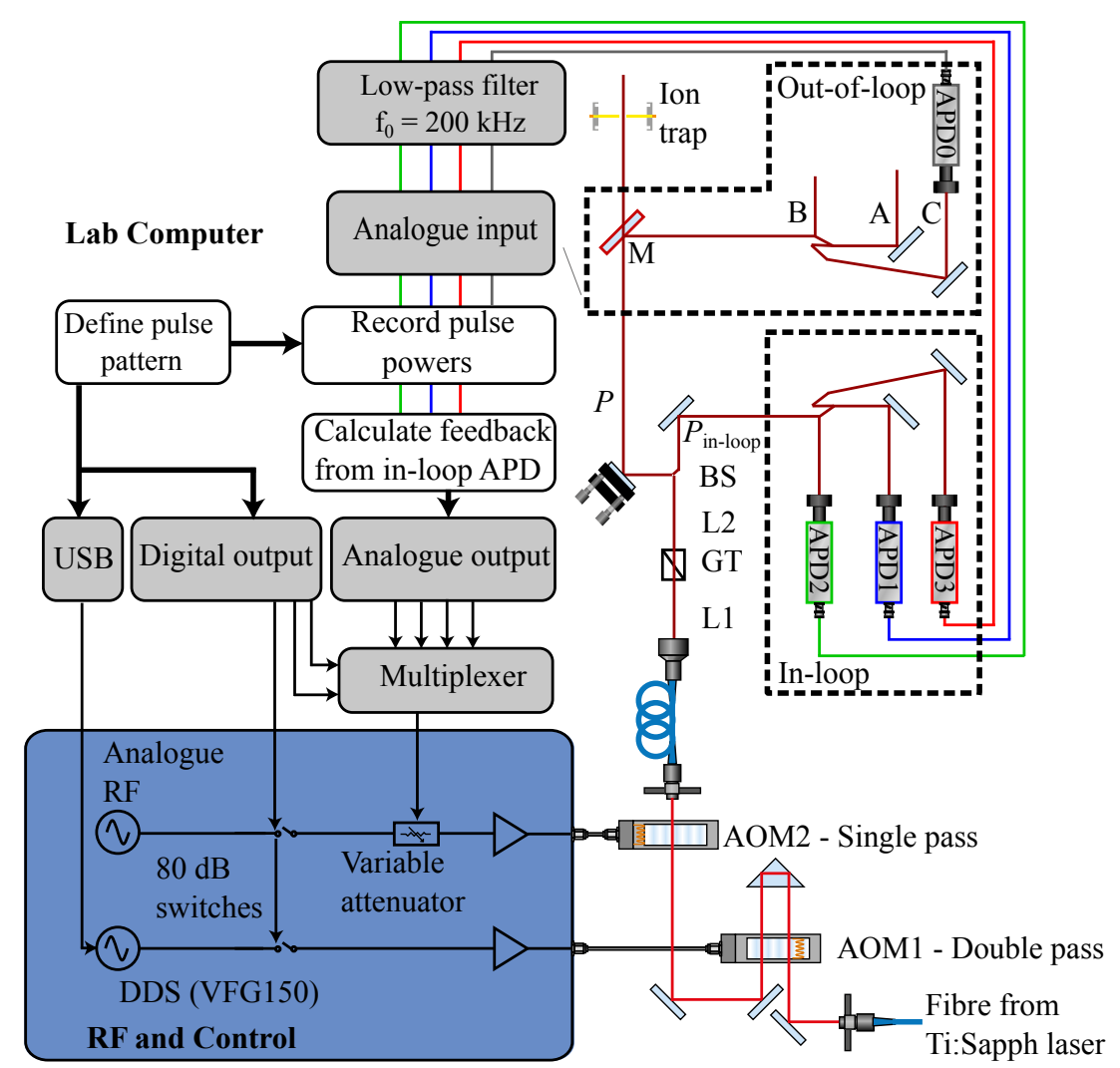

Fig. 1 Schematic of hardware used to stabilise the pulse powers. Measurements on APDs 1-3 are used to calculate the feedback. APD0 measures the power of the transmitted beam (position A), the first reflected beam (position B) or second reflected beam (position C) arising from a second wedged optic. In each position, APD0 measures approximately the same power as the corresponding in-loop APD. In coherent control experiments, the mirror highlighted in red (M) is removed and the beam with power $P$ is incident on the ion. GT; Glan-Taylor polariser, BS; non-polarising 50:50 beam splitter. Lens L1 collimates light from the fibre and lens L2 focuses the beam to a waist at the ion trap center.

or APD3 and APD0, has a signal-to-noise ratio $>100$ and is not saturated. These extremes of power cover the range of Rabi frequencies required for coherent control as well as for spectroscopy at the highest resolution. High powers are principally for coherent control of the ion's motional states, but are also used for state preparation via resolved sideband cooling. Although the latter is less sensitive to intensity fluctuations, stable operation is advantageous for minimising state preparation errors
[11]. Mid-range powers are required for carrier transition spectroscopy and electronic state control. The lowest powers are for the longest pulse durations necessary to realise high spectroscopic resolution and measurement accuracy. In both Rabi and Ramsey spectroscopy, pulses of longer duration result in a spectrally narrower envelope, thus minimising errors associated with off-resonant excitation of nearby transitions. Additionally, long duration pulses of low intensity minimise the ac-Stark shift 
of the qubit transition caused by the interaction of the laser with off-resonant transitions.

The Hamamatsu C10508-01 APDs were chosen for their high bandwidth $(10 \mathrm{MHz})$ to measure short $(\sim 10 \mu \mathrm{s})$ pulses, as well as for their radiometric stability. When operated at the lowest gain setting, we find the measured performance of this APD is comparable to Hamamatsu S-1337 PIN photodiodes which are commonly used for radiometric applications [24]. As the APDs are used for accurate scaling of Rabi frequencies (via optical power), it is required that the linearity of the detectors themselves is well known. This was assessed using the method of Theocharous $[25,26]$, with a minimum linearity factor of 0.998 measured across the dynamic range of the detector. The spatial uniformity of the APDs was also measured [27], with maximum variations in quantum efficiency of $2 \%$ across the full active area (1 $\mathrm{mm}$ diameter) of the detector, when measured on $20 \mu \mathrm{m}^{2}$ pixels. Minimising the sensitivity of the detected signal to beam drift is a compromise between averaging over efficiency variations and minimising signal loss at the edge of the beam. A beam diameter of $100 \mu \mathrm{m}$ on the detector surface was chosen. A $100 \mu \mathrm{m}$ spatial drift of this beam across the surface during a measurement would cause relative fluctuations in the measured signal with a standard deviation of $\leq 10^{-4}$. An optical bandpass filter (Semrock FF02-675/67-25) at the input of each APD eliminates ambient light, and any residual $1064 \mathrm{~nm}$ and
$532 \mathrm{~nm}$ emanating from the titanium-sapphire laser, to well below the noise floor of the detector.

The entire pulse sequence, as measured on each APD, is sampled at $400 \mathrm{kHz}$ using a high speed analogue input card (NI PXI-6254, 16 bit resolution). To prevent noise above the sampling frequency being down-sampled and projected onto the error signal, the APD signals are electronically filtered. Passive low-pass RC filters have an insufficiently steep roll-off, so each APD channel contains an active fourth-order Bessel filter (Maxim MAX275ACPP+) with a $3 \mathrm{~dB}$ cut-off frequency of $200 \mathrm{kHz}$, thus enabling detection of pulses down to $10 \mu \mathrm{s}$. We note that in the case of bichromatic laser fields for entanglement $[10,28]$, the power incident on the photodiodes oscillates at $\geq 10$ times the filter cut-off frequency; hence the mean power of the amplitude-modulated light field will be recorded in the sampling process. To avoid instabilities arising in that case, the pulse duration should be matched precisely to an integer number of the bichromatic beat period, which is feasible with the DDS source. Independent correction signals that stabilise the pulse powers are generated by an analogue output card (NI PXI-6733) and are applied to the variable attenuator via a multiplexer switch. The TTL signals used to control this switch, as well as ones used to control the RF switches, are generated by a high speed digital I/O card (NI-PXIe-6537). Opto-couplers in each TTL line provide electronic isolation between the instrumentation and the digital I/O card. Instrumentation amplifiers provide iso- 
lation from ground noise on the analogue output card through common-mode rejection.

\subsection{Power calibration}

The optical power $P$ of each pulse may be set in the range $300 \mathrm{nW} \leq P \leq 5.2 \mathrm{~mW}$. For a given detector, the absolute Rabi frequency $\Omega$, achieved with power $P$ at an APD stabilisation setpoint $V$, can be measured directly with the ion. Relying on the detector's linearity, an arbitrary Rabi frequency $\Omega_{a r b}$ can be set accurately and precisely via a scaled setpoint $V_{a r b}$ for power $P_{a r b}$ (within the range of the detector), without the need to measure $\Omega_{a r b}$ directly. The AOM system's response is calibrated to permit simple and rapid initialisation of the power to within $2 \%$ of the desired power $P_{a r b}$, so that the feedback locks on to the setpoint $V_{\text {arb }}$ in a minimum number of measurement cycles. The use of three cascaded APDs increases the range of applied RF powers, and subsequent optical powers, for which the signalto-noise is sufficient to give accurate calibration.

We verify the calibration routine by programming a linear ramp in the set optical power at the ion $\left(P_{\text {set }}\right)$, as a fraction of the maximum possible value $\left(P_{\max }\right)$, and measuring the arising signals on APD1, APD2 and APD3 (see Fig. 2(a)). While $P_{\max }$ is measured by an optical power meter, $P_{\text {set }}$ is a programmed value implemented via the calibration [23]. In Fig. 2(b), the data from the APD with the best signal-to-noise ratio at each value of $P_{\text {set }}$ is normalised and combined to give the total lin-
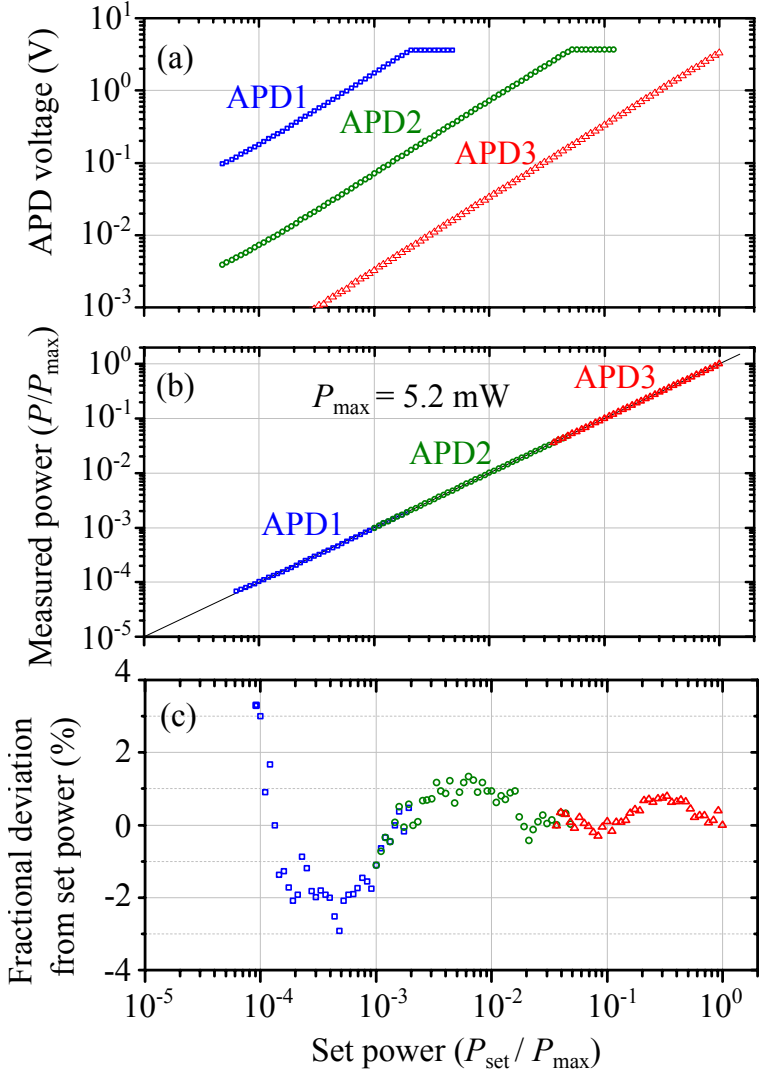

Fig. 2 (a) Measured voltage on APDs1-3 as a function of $P_{\text {set }} / P_{\max }$. A single optical pulse of $1 \mathrm{~ms}$ duration was used to record each data point; consecutive pulses in the series were recorded at a rate of $113 \mathrm{~Hz}$. In (b) the same data is normalised and combined to give the total linearised response of the system. The black solid line is the ideal linear response of the system. Finally, (c) shows the fractional deviation from this ideal response. Errors in the data are less than the systematic deviations in (c), and are estimated from the spread of adjacent data points in (c) to be $\leq \pm 0.5 \%$.

earised response of the system, which overlaps well with the ideal response of unity gradient and zero intercept. Figure 2(c) shows the fractional deviation of the data from the ideal set power, with an error of $<2 \%$ observed for $P_{\text {set }} / P_{\max }>10^{-4}$. The systematic deviations visible 
in Fig. 2(c) could be minimised; however, the power initialisation is already sufficiently accurate for the stabilisation scheme to eliminate the offset. In the context of coherent rotations of atomic qubits, the exact set-point of power is determined by a direct measurement of the ion-laser coupling strength (i.e. the Rabi frequency).

\subsection{Power stabilisation system - concept and results}

Qubit gate operations are performed by a sequence of pulses with various durations, powers and frequency detunings. For accurate readout of the qubit's final state, this sequence is repeated many times. The feedback principle relies on the measured powers of pulses in each sequence to inform corrections to the AOM settings, in order to achieve the desired powers for each pulse in the subsequent sequence. The linearised response of the AOM (Fig. 2(b)) ensures rapid locking to the desired setpoints. The stabilisation system reported here reduces power variations in pulse sequences up to Fourier frequencies of $\sim 50 \mathrm{~Hz}$. Intensity fluctuations on a timescale that is short compared to the pulse sequence duration (typically $<5 \mathrm{~ms}$ ) are not compensated for. A measurement of the passive power stability of the titaniumsapphire laser shows three dominant noise components at discrete Fourier frequencies in the range $50 \mathrm{~Hz}-100 \mathrm{kHz}$. Each component has a fractional power noise of $<8 \times$ $10^{-4}$. Following the analysis presented in section 4 , no feedback in this frequency range is required in order to meet the target infidelity. However, if an alternative laser source exhibits high-frequency power noise, then it would be simplest to correct for this noise before the light enters the pulse shaping AOMs and intensity stabilisation system presented here (i.e. where the laser beam is continuous, before input to the delivery fibre in the bottom right-hand side of Fig. 1).

The principle of the pulse-power feedback loop for a defined pulse sequence (see Fig. 3) is as follows. In the $j^{\text {th }}$ repeated sequence, the $i^{\text {th }}$ pulse has a duration $T_{i}$ (typically $5 \mathrm{~ms} \geq T_{i} \geq 5 \mu \mathrm{s}$ ), and a power $P_{i}$ at the ion (where $300 \mathrm{nW} \leq P_{i} \leq 5.2 \mathrm{~mW}$ ). This sequence is communicated to the DDS via USB 2.0 and is triggered at a frequency of $113 \mathrm{~Hz}$, which is representative of measurement cycles with trapped ions and is asynchronous with harmonics of the $50 \mathrm{~Hz}$ mains frequency. The samples acquired during the $i^{\text {th }}$ optical pulse in the $j^{t h}$ sequence are averaged to give a measured voltage value $V_{i, j}$. Similarly, samples acquired in a $100 \mu$ s window after the optical pulses are averaged to give a dark level $D_{j}$ for each sequence. To stabilise the power of the $i^{\text {th }}$ pulse to a user-defined set-point $S_{i}$, a correction signal $C_{i, n}$, applied to the variable attenuator during the $i^{\text {th }}$ pulse in the $n^{\text {th }}$ sequence, is calculated according to

$$
C_{i, n}=V_{\text {off }}-G_{i} \sum_{j=n-N}^{n-1}\left(\frac{\left(V_{i, j}-D_{j}\right)-S_{i}}{N}\right)
$$

Here, $V_{\text {off }}$ is a fixed offset from zero volts that determines the initial attenuation setting, $G_{i}$ is a positive gain factor set independently for each pulse in the sequence, and $N$ 


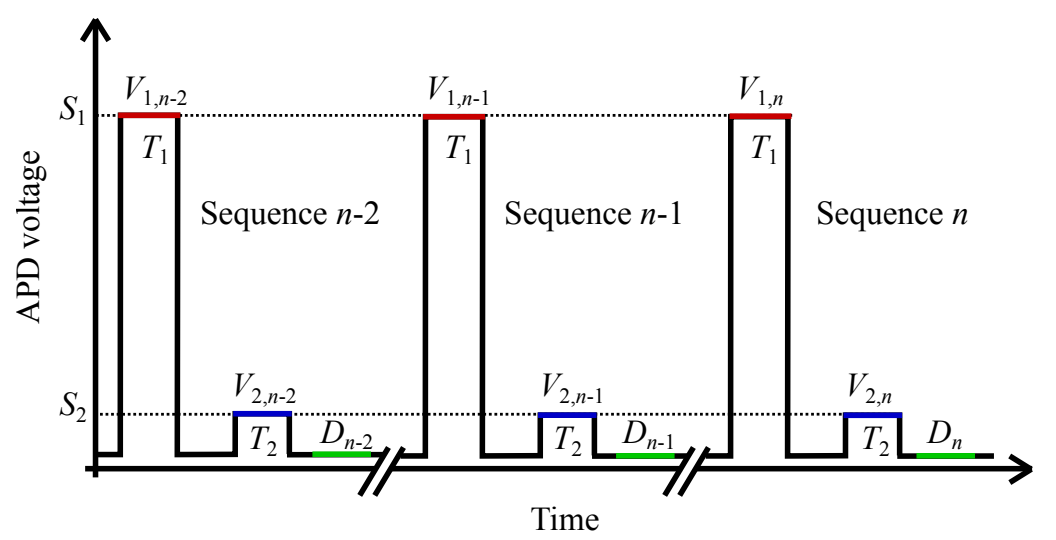

Fig. 3 Schematic showing three consecutive pulse sequences $(j=n-2, n-1, n)$, each containing two pulses $(i=1,2)$ of duration $T_{1}$ and $T_{2}$, which are sampled at a rate of $400 \mathrm{kHz}$ by one of the in-loop APDs. Within one sequence, samples highlighted in red and blue are averaged to give the values $V_{1, j}$ and $V_{2, j}$, which are stabilised to set-points $S_{1}$ and $S_{2}$ respectively. Similarly, samples highlighted in green are averaged to give the dark reading $D_{j}$ for each sequence.

is the number of pulses that are averaged over to calculate the feedback. Stabilising to one pulse only does not replicate the same degree of instability in pulses of differing powers; this is due to drifts in the AOM's optical response. Hence the correction signals are multiplexed in time to enable independent control of dissimilar pulses.

We assessed the performance of the pulse power stabilisation system using a sequence of two pulses, firstly with APD3 as the detector from which the feedback is calculated. The pulse durations were $T_{1}=T_{2}=100 \mu \mathrm{s}$, separated in time by $100 \mu \mathrm{s}$, and were stabilised to setpoints $S_{1}=3.3 \mathrm{~V}$ and $S_{2}=0.12 \mathrm{~V}$. The former value is close to the saturation voltage of the APD, and the latter is the value where the signal-to-noise drops below 100. Therefore in this example, $S_{1}$ and $S_{2}$ span the useful operating range of the APD. From the system response calibration and linearisation (see Fig. 2), these set points relate to powers at the ion of $P_{1}=5.17 \mathrm{~mW}$ and $P_{2}=196 \mu \mathrm{W}$. Averaging over $N=2$ pulses and setting the gain for each pulse to $G_{i}=6 / S_{i}$ optimises the feedback for power stability. Furthermore, by choosing $V_{\text {off }}=8 \mathrm{~V}$, the variable attenuator is in a regime where its response is approximately linear. AOM2 is configured to operate a few percent below its maximum diffraction efficiency to provide sufficient range for stabilisation. In a similar fashion, lower power ranges were evaluated with the feedback via APD2 or APD1.

A limitation to the present implementation of the system is that if any of the APDs are subject to an optical power of more than approximately twice the saturation power of $9 \mu \mathrm{W}$, measurements of subsequent optical pulses in the sequence are distorted. After saturation, the APD voltage is negative and recovers to its accurate steady state in a double exponential decay (time 
constants $85 \mu$ s and $9 \mathrm{~ms}$ ) with amplitudes dependent on saturating pulse power. Therefore, sufficient recovery time is required in instances where an earlier higher power pulse saturates an APD which is relied upon by a subsequent lower power pulse for accurate stabilisation. To eliminate this constraint, optical modulators could be used to ensure detector illumination by non-saturating pulses only.

Figure 4(a) shows a long-term measurement of the fractional variation in pulse power (averaged over 1 second intervals) for pulse 1 with the stabilisation system engaged. Data from both the in-loop (red, APD3) and out-of-loop (grey, APD0-C) detectors are presented. Similarly, Fig. 4(b) shows the time series for pulse 2. In both cases there is some relative drift $\left(\sim 1 \times 10^{-3}\right.$ over $\left.\sim 10^{5} \mathrm{~s}\right)$ between the in- and out-of-loop signals over the duration of the measurement. We observe correlations between measured laboratory humidity and the data, suggesting that this causes variations in the reflectivity of the beamsplitter optical coating. This effect could be reduced by using a beamsplitter coating created by ion-beam sputtering, rather than by physical vapour deposition as per the optics used in this experiment. For all the data presented in this work, the ambient laboratory temperature was stable to within $\pm 0.1 \mathrm{~K}$. This corresponds to changes in surface reflectivity of $\leq 4 \times 10^{-6}$ for the optical materials used (fused silica and N-BK7, thermooptic coefficients $\left.d n / d T<10^{-5} \mathrm{~K}^{-1}\right)[29,30]$, rendering such effects negligible. The intrinsic electronic drift of the Bessel filter and analogue input card was measured to be negligible compared to drift level shown in Fig. 4. Drifts in the dark current of each APD are accounted for by the measurement of $D_{n}$ in every sequence. Small alignment drifts of the beam across each APD surface of non-uniform quantum efficiency are expected to contribute relative signal fluctuations of $\leq 10^{-4}$. The APD module contains a microcontroller to compensate temperature-induced fluctuations in gain, and an experimental comparison with an unbiased Hamamatsu S-1337 PIN photodiode shows agreement to $\leq 4 \times 10^{-4}$.

In order to quantify the instability of the system on varying time-scales, we use the data presented in Fig. 4 to calculate the 2-sample fractional Allan deviation [31], as shown in Fig. 5(a). The Allan deviations $\sigma_{P}$ as a function of averaging time $\tau$ for the in-loop measurements are shown as solid and dashed red lines for pulse 1 and 2 respectively. Similarly, out-of-loop measurements are shown in grey. The experimental run during which this data was acquired is denoted $\Gamma$. To show effective stabilisation over the full dynamic range of the system, two further experimental runs, similar to $\Gamma$, were performed. In experiment $\Delta(\Lambda)$, APD2(APD1) and APD0 in position $\mathrm{B}(\mathrm{A})$ were used as the in- and out-of-loop detectors respectively. The corresponding Allan deviations for $\Delta$ and $\Lambda$ are given in Fig. 5(b,c). In Fig. 5(d), the power of each pulse at the ion is shown on a logarithmic scale. As the ranges of the measurements overlap, it is clear that the pulse power stabilisation system can be effectively 

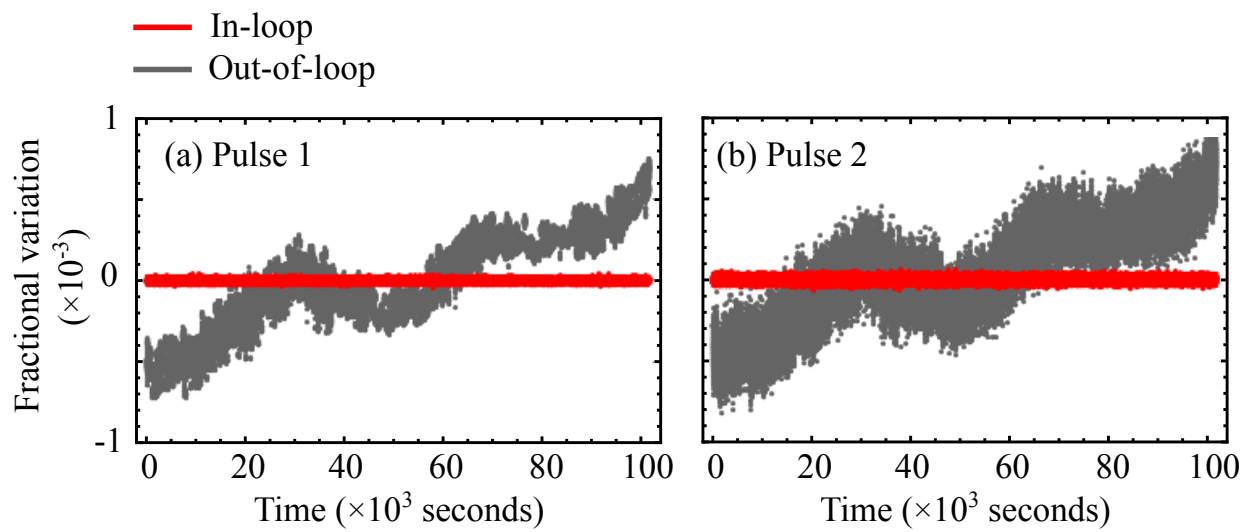

Fig. 4 (a) and (b) show the fractional variation in pulse power (relative to the mean) over 1 second intervals, as measured on the in-loop (red - APD3) and out-of-loop (grey - APD0 in position C) detectors, for pulse 1 and 2 respectively. The lower signal-to-noise ratio when measuring pulse 2 is evident in the greater noise amplitude in (b). Here the pulse powers at the ion are $P_{1}=5.17 \mathrm{~mW}$ and $P_{2}=196 \mu \mathrm{W}$.

utilised over the full dynamic range of the system, and the out-of-loop fractional Allan deviation is $<2.2 \times 10^{-4}$ for all averaging times from 1 to 16384 seconds for all three experiments. It is informative to compare these results to the scenario whereby the single-ion response is used as the stabilisation discriminant; i.e. measuring excitation probability for a $\pi / 2$ pulse. Based on the quantum projection noise limit, and neglecting all other systematic effects, a $2 \mathrm{~ms}$ interrogation cycle results in a fractional instability of power at $10^{-4}$ only after $\sim 2$ hrs of averaging. While a linear improvement could be realised using a $(2 n+1) \pi / 2$ pulse, it remains orders of magnitude slower than our approach. In section 4, the implications of the measured levels of instability are discussed in the context of high-fidelity quantum gate operations.

\section{Beam pointing stabilisation}

The aforementioned pulse power stabilisation scheme can operate concurrently with an independent feedback system for stabilising the focused laser beam position to the ion trap center. This is based on a quadrant photodiode (QPD) onto which the beam waist at the trap center is imaged. The apparatus and principles of the feedback loop, as well as results which characterise the long-term instability of the system, are presented in this section.

\subsection{Beam position stabilisation system - experimental setup}

The beam that coherently controls the trapped ion is transmitted through the vacuum chamber and incident on a beamsplitter (see Fig. 6). Each subsequent beam is imaged onto an independent QPD (ThorLabs PDQ80A). Each QPD produces two difference signals correspond- 

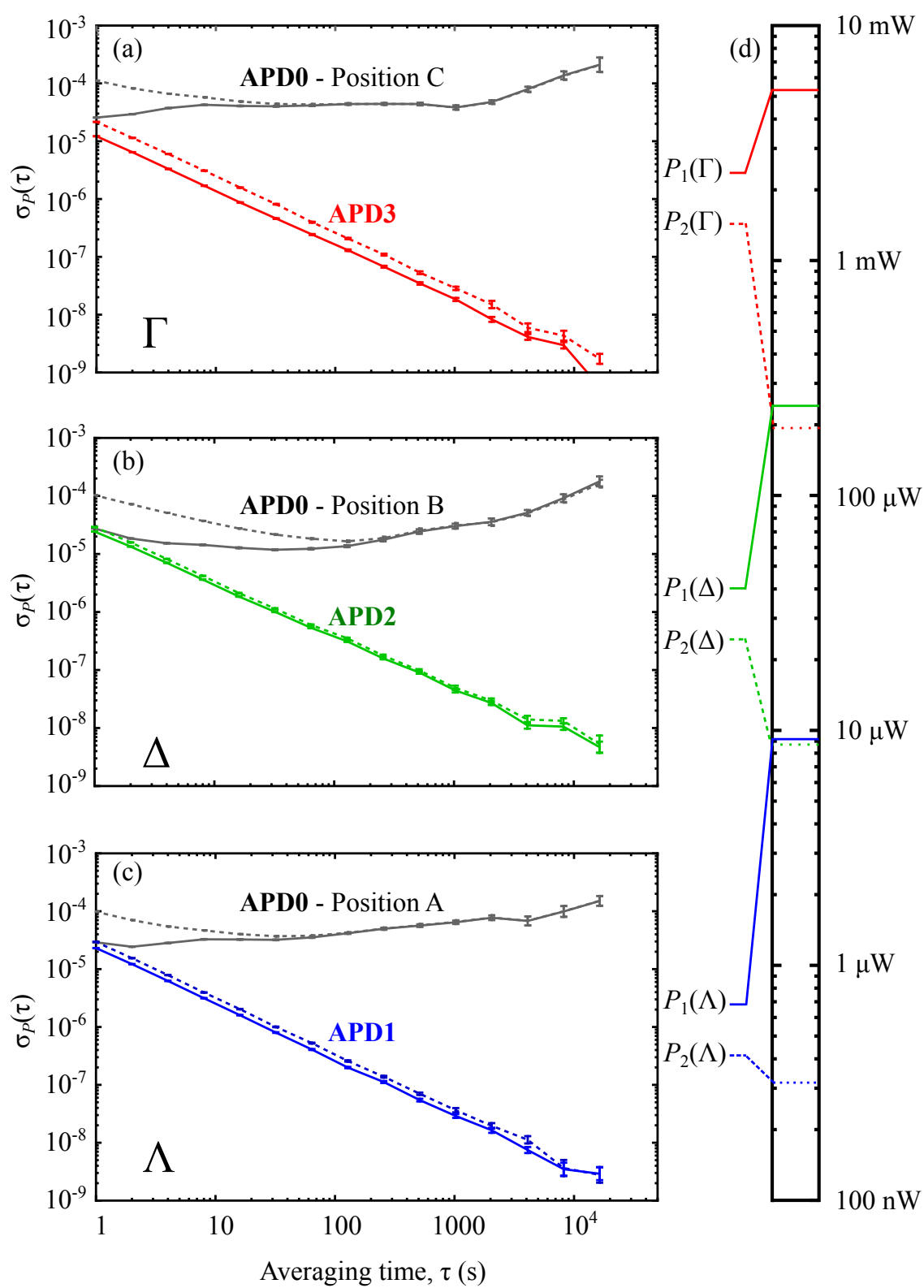

$100 \mu \mathrm{W}$

$P_{1}(\Delta)$ $P_{2}(\Delta)$

Fig. 5 (a-c) show the Allan deviation of in-loop (colored) and out-of-loop (grey) measurements for pulse 1 (solid) and pulse 2 (dashed) in experiments $\Gamma, \Delta$ and $\Lambda$ respectively. The power at the ion for each pulse is shown on a logarithmic power scale in (d). The range of each experiment overlaps, showing that the stabilisation can be effectively utilised over the full range of the system.

ing to the horizontal $\left(x_{\text {diff }}\right)$ and vertical $\left(y_{\text {diff }}\right)$ displacements in the laboratory frame. These are measured by the analogue input card and, by means of external calibration factors $\alpha_{x}$ and $\alpha_{y}$, are used to infer the position of the beam focus in the plane of the ion. QPD1 oper- ates as the in-loop detector from which a discriminant is derived, whereas QPD2 serves as an out-of-loop monitor.

Beam pointing is controlled by a piezo-actuated mirror (New Focus Picomotor 8885), with an angular resolution of $1.5 \mu \mathrm{rad}$, corresponding to a step size of $180 \mathrm{~nm}$ 


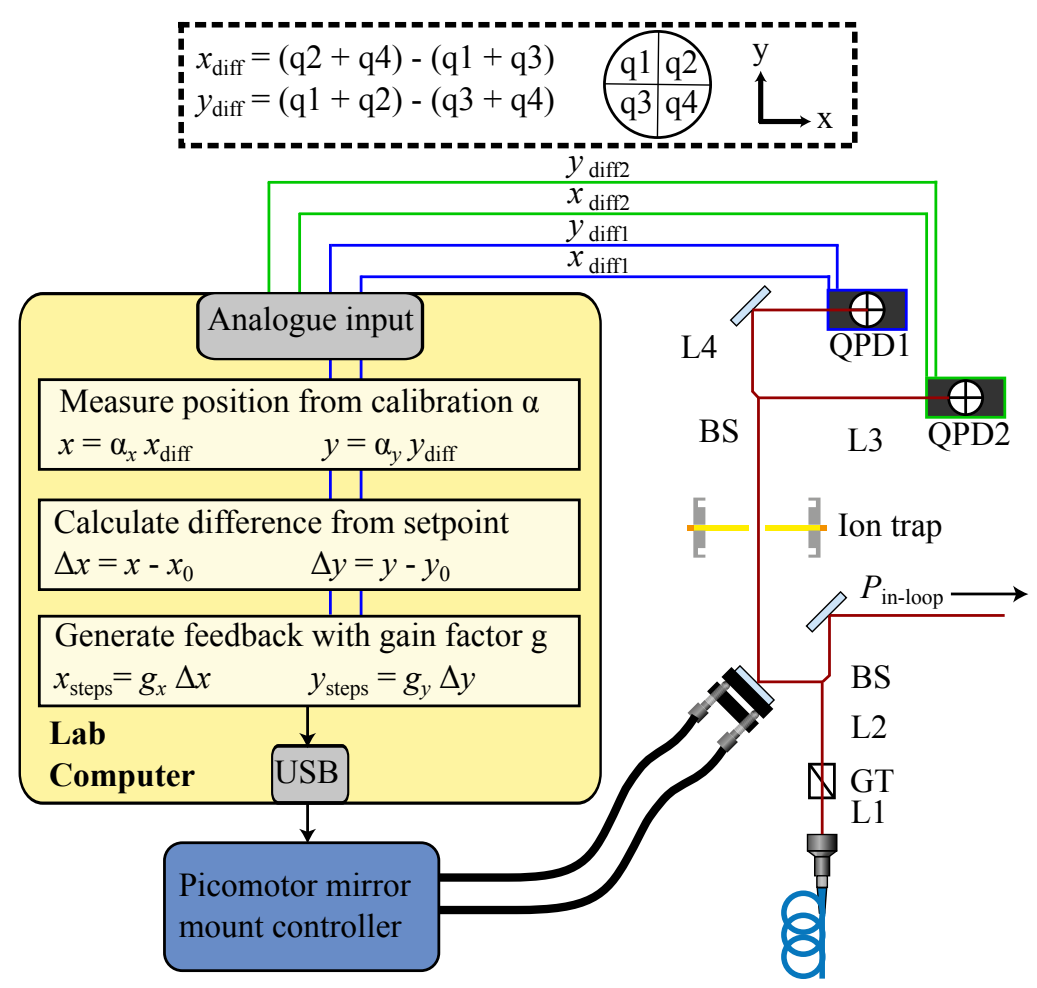

Fig. 6 Schematic of hardware used in a feedback loop to stabilise position of the beam waist. Lenses focus the beam to a waist $2 w_{0}=45 \mu \mathrm{m}$ at the trap center, and image this waist onto the QPDs. Measurements of the difference signals $x_{\mathrm{diff}}$ and $y_{\text {diff }}$ on QPD1 are used to generate the feedback signal for the Picomotor mirror mount. QPD2 performs an out-of-loop measurement of the beam position. GT; Glan-Taylor polariser, BS; 50:50 beam splitter, L1; multi-element $f=29$ mm, L2; achromatic doublet $f=200 \mathrm{~mm}$, L3 and L4; achromatic doublet $f=100 \mathrm{~mm}$.

at the ion. When combined with a translation stage to set the longitudinal position of the focusing lens, this enables precise positioning of the beam waist inside the vacuum chamber. Repeatable beam positioning is achieved with QPD1 as a spatial reference, thus circumventing hysteresis of the Picomotor. Since the laser beam originates from a single-mode fibre, contributions to the QPD signals from instabilities in the transverse mode profile of the beam are considered negligible. We note that a commercial system [32] promises pointing stability suited to this application (i.e. servo loop accuracy quoted as
$<1 \mu \mathrm{m},<1 \mu \mathrm{rad})$. However, servo loop accuracy alone is insufficient for the purposes of this work, since an outof-loop Allan deviation measurement is nevertheless required to validate the performance of beam pointing stabilisation [33]. This measurement is detailed in section 3.2.

3.2 Beam position stabilisation system - concept and results

For stabilisation, the difference between the co-ordinates of the measured beam position $(x, y)$ and a user-defined 
set-point $\left(x_{0}, y_{0}\right)$ form a discriminant (see yellow shaded box, Fig. 6). A feedback correction is derived in terms of number of Picomotor steps $x_{\text {steps }}$ and $y_{\text {steps }}$, via userdefined gain settings $g_{x}$ and $g_{y}$. Using proportional feedback only, the system stabilises the measured position to the chosen setpoint within the limits of the Picomotor angular resolution.

Signals arising from a position-stabilised beam incident on QPD1 and QPD2 were recorded for 35 hours over 1 second intervals; optimised feedback was achieved with $g_{x}=g_{y}=0.6$. The resulting time series for the in- and out-of-loop measurements for $r=\sqrt{x^{2}+y^{2}}$, as well as the corresponding absolute Allan deviations, are shown in Fig. 7; this shows that $\sigma_{r}<0.1 \mu \mathrm{m}$ for all averaging times. Some disagreement between the in- and out-of-loop measurements is evident, possibly due to differential drift of the opto-mechanical components in the separate optical paths after the beamsplitter. However, for a typical spot size of $2 w_{0}=45 \mu \mathrm{m}$ as used in our experiment, the peak-to-peak drift as measured on both QPDs is $<1 \%$ of the beam diameter.

For comparison, data showing passive stability of the opto-mechanical system is also presented in Fig. 7(a). This demonstrates that in practice, it will suffice to engage the position stabilisation feedback for a few seconds duration at intervals of approximately 1 hour (see Fig. $7(\mathrm{~b}))$. In our laboratory the beam path is well-shielded from air currents arising from air conditioning, which can distort beam pointing, but in other laboratory en- vironments this may not be the case [34]. The method presented here can of course be used at much shorter intervals (e.g. $\sim 1$ s) to monitor and compensate beam drift in less benign environments. It is straightforward to interleave such a procedure between the acquisition of complete data sets for coherent control routines. The system does not compensate for positional drifts of the ion trap electrode structure with respect to the optical components used for stabilisation. However in our apparatus, differential movement between the ion trap chip [35] and the compact vacuum chamber [36] is eliminated as they are bonded directly together; the chamber and a compact opto-mechanical setup are mounted directly onto a single breadboard. With no detectable drift of the fluorescence images of laser-cooled ions, we expect trapto-beam differential drifts to be no greater than those shown in Fig. 7(a).

\section{Estimates of intensity-induced contributions to trapped-ion quantum gate infidelity}

The principal aim of this system is to achieve an intensity instability which is low enough that this parameter alone contributes $\leq 10^{-4}$ to the overall infidelity of a quantum gate. To determine the viability of meeting this criterion, the measured instabilities in laser power and beam pointing were used to estimate the associated contribution to infidelity for two laser-driven quantum gate operations, namely the Pauli X and Mølmer-Sørensen entangling gates. 

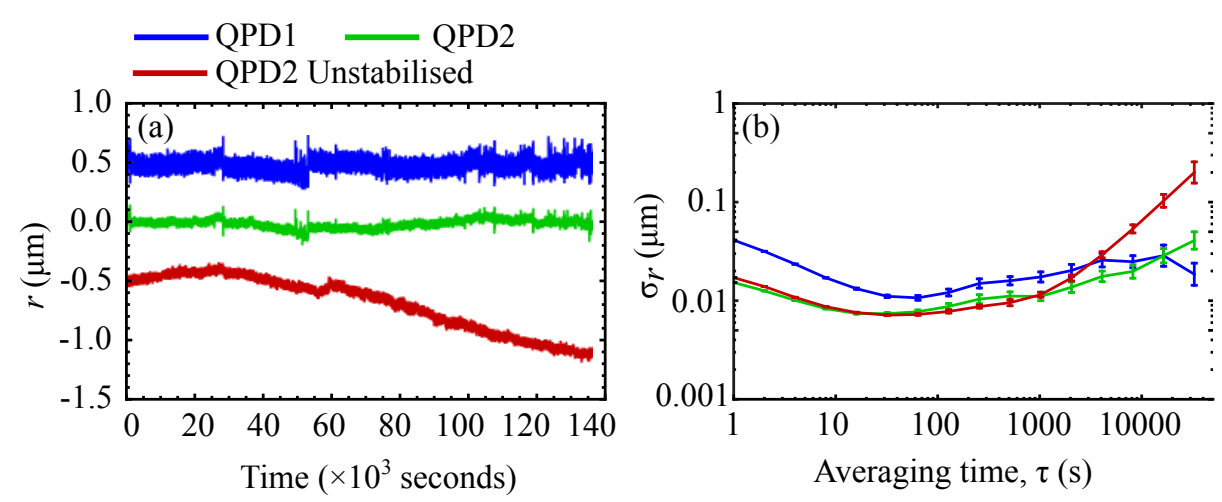

Fig. 7 (a) Deviation of the beam position from its original value as detected on the in-loop (blue, QPD1) and out-of-loop (green, QPD2) detectors with the feedback system engaged. Beam position data recorded with the unstabilised, free-running system is shown for comparison (red). Data sets are offset vertically for clarity. (b) Corresponding Allan deviation of the data shown in (a), showing a close agreement between the two measurements over all averaging times.

We consider ions with internal ground and excited states $|g\rangle$ and $|e\rangle$ respectively, which are coupled to a (near-) resonant optical pulse with Rabi frequency $\Omega$. A Pauli X-gate acts on a single ion in an initial state $\left|\Psi_{i}\right\rangle=a|g\rangle+b e^{i \beta}|e\rangle$ to produce a target state $\left|\Psi_{t}\right\rangle=$ $b e^{i \beta}|g\rangle+a|e\rangle$. The fidelity of the operation is $F_{x}=$ $\left|\left\langle\Psi_{t}|U(t)| \Psi_{i}\right\rangle\right|^{2}$, where

$$
U(t)=\left(\begin{array}{cc}
\cos (\Omega t / 2) & -i e^{i \phi} \sin (\Omega t / 2) \\
-i e^{i \phi} \sin (\Omega t / 2) & \cos (\Omega t / 2)
\end{array}\right)
$$

represents the interaction of the optical pulse of phase $\phi$ with an ion for duration $t$. Expanding and simplifying $F_{x}$ gives

$$
\begin{array}{r}
F_{x}=\frac{1}{2}\left[a^{4}+4 a^{2} b^{2}+b^{4}-\left(a^{4}+b^{4}\right) \cos (\Omega t)\right. \\
\left.+2 a^{2} b^{2} \cos (2 \beta)(1+\cos (\Omega t))\right],
\end{array}
$$

which is unity when the desired Rabi frequency $\Omega_{0}=$ $\pi / t$. In the event of a small error $\delta \Omega=\Omega-\Omega_{0}$, a Taylor expansion of $F_{x}(\Omega)$ shows that the minimum fidelity $F_{x, \min }$ occurs for $\left|\Psi_{i}\right\rangle=|g\rangle,|e\rangle$ and $[\sin \theta|g\rangle+$ $\left.e^{i(n+1 / 2) \pi} \cos \theta|e\rangle\right]$. In this case, the maximum infidelity due to Rabi frequency error, $\left(1-F_{x, \min }\right)_{\Omega}$, is

$$
\left(1-F_{x, \min }\right)_{\Omega} \approx(\pi / 2)^{2}(\delta \Omega / \Omega)^{2}
$$

When a two-ion string is initialised in the ground state $|g g\rangle$ and subject to the Mølmer-Sørensen entangling gate [37], the maximally entangled state $\left|\Psi_{\max }\right\rangle=$ $(1 / \sqrt{2})[|g g\rangle-i|e e\rangle]$ is produced with fidelity $F_{M S}=$ $\left\langle\Psi_{\max }|\rho| \Psi_{\max }\right\rangle=\left(\rho_{g g, g g}+\rho_{e e, e e}\right) / 2+\operatorname{Im} \rho_{e e, g g}[10,38$, 39]. $\left|\Psi_{\max }\right\rangle$ is created when the optical pulse duration $T=2 \pi / \epsilon$, where $\epsilon$ is the gate detuning [10]. Under this condition, and noting earlier theoretical investigation [38], we calculate the gate fidelity for ions cooled to the motional ground state to be

$$
F_{M S}=\frac{1}{2}+\frac{1}{2} \sin \left(\frac{2 \pi \Omega^{2} \eta^{2}}{\epsilon^{2}}\right)
$$

where $\eta$ is the Lamb-Dicke parameter (a measure of the extent of the ion's ground-state wavefunction),

$$
\eta=k\left(\frac{\hbar}{2 m \omega}\right)^{1 / 2}
$$


with $k$ being the laser wavevector, and $m$ and $\omega$ the ion's mass and motional frequency respectively. For a small fractional error $\delta \Omega / \Omega$, the associated contribution to infidelity (derived by a Taylor expansion of equation 5 about $\left.F_{M S}\left(\Omega=\Omega_{0}\right)=1\right)$, is given by

$$
\left(1-F_{M S}\right)_{\Omega} \approx(\pi / 2)^{2}(\delta \Omega / \Omega)^{2}
$$

which is consistent with $[4,10]$.

In the two examples just described, the infidelities $\left(1-F_{M S}\right)_{\Omega}$ and $\left(1-F_{x, \text { min }}\right)_{\Omega}$ have the same dependence on Rabi frequency error. However, the measured physical parameters are instabilities in beam power and position.

For an optical qubit, $\Omega \propto \sqrt{P}$ and equation 4 can be recast in $P$ to give the contribution to infidelity from a fractional error in pulse power $\delta P / P$ as

$$
\left(1-F_{x, \min }\right)_{P} \approx(\pi / 4)^{2}(\delta P / P)^{2}
$$

with $\left(1-F_{M S}\right)_{P}$ having the same dependence.

Concerning the contribution to infidelity from small fluctuations $\delta r$ in beam position, we consider first the single-qubit gate in an experimentally realistic scenario where the focused beam is imperfectly centered on the ion. It is assumed that the ion sits at an offset $r_{1}$ from the center of a Gaussian beam $\left(r_{1} \ll w_{0}\right)$, and that the pulse power and duration are optimised for maximum fidelity. Noting that $\Omega$ has a Gaussian dependence on $r$, equation 4 can be rewritten in terms of $r$, to give

$$
\left(1-F_{x, \min }\right)_{r} \approx \pi^{2}\left(r_{1} \delta r / w_{0}^{2}\right)^{2} .
$$

For the beam diameter $2 w_{0}=45 \mu \mathrm{m}$ used in this apparatus, the beam can be positioned easily such that the ion sits well within $2 \mu \mathrm{m}$ of beam centre. This is achieved by performing pulsed-probe spectroscopy as the beam is raster-scanned across the ion.

Based on equations 8 and 9, Fig. 8 shows the projected Allan deviation in infidelity $\sigma_{\left(1-F_{x, m i n}\right)}$ arising from the measured instabilities in power and position of the laser beam alone. The contribution from power variations is calculated by setting the values of $\sigma_{P}$ from data set $P_{2}(\Gamma)$ (which has the highest average value of those shown in Fig. 5) as $\delta P / P$ in equation 8. Similarly the contribution from beam position fluctuations are calculated by setting the out-of-loop values of $\sigma_{r}$ in Fig. 7(b) as $\delta r$ in equation 9 at an offset $r_{1}=2 \mu \mathrm{m}$ in a beam of $2 w_{0}=45 \mu \mathrm{m}$. Figure 8 shows that the projected deviation in $\left(1-F_{x, \min }\right)$ is orders of magnitude below the $10^{-4}$ threshold (as discussed in the introduction) for all experimental averaging times.

For the Mølmer-Sørensen two-qubit gate, the dependence of $\left(1-F_{M S}\right)$ on $\Omega$ can only be considered identical to that for $\left(1-F_{x, \min }\right)$ (as per equations 4 and 7 ), when both ions are illuminated equally; i.e. $\Omega_{1}=\Omega_{2}$, where the subscript denotes the individual ions. In that case, the contributions to infidelity would be identical to those in Fig. 8. Experimentally, setting $\Omega_{1}=\Omega_{2}$ will require engaging the power stabilisation and measuring the Rabi frequencies on both ions at different beam positions. 


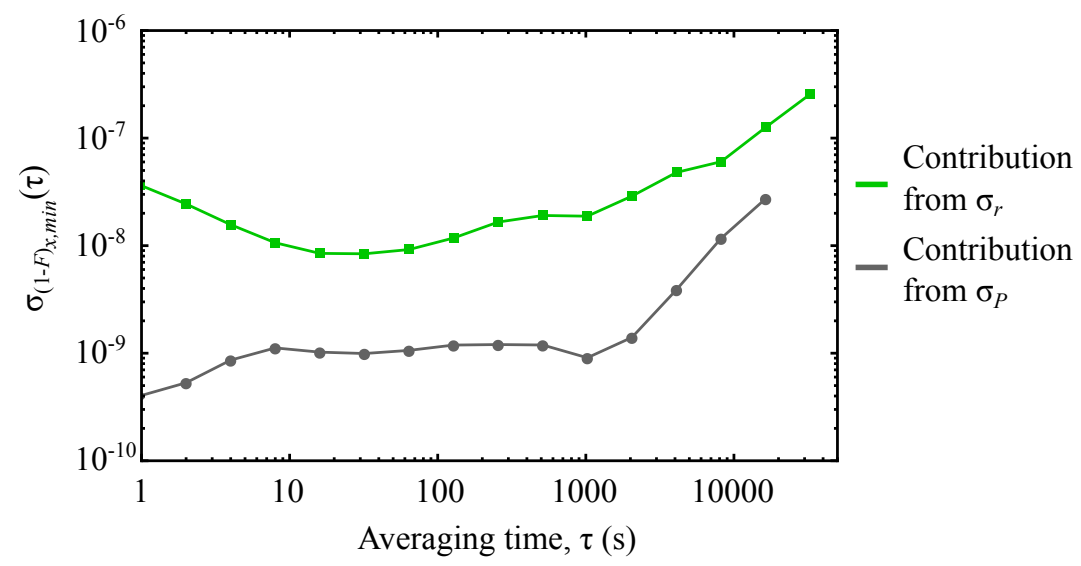

Fig. 8 Projected Allan deviation of the contributions to single-qubit infidelity, $\sigma_{\left(1-F_{x, m i n}\right)}$, due to beam power (grey) and position (green) instabilities. Calculations assume a laser-driven optical qubit transition and use measurements of $\sigma_{P}$ and $\sigma_{r}$. The contribution from $\sigma_{r}$ is plotted for a beam of $2 w_{0}=45 \mu \mathrm{m}$ with an ion offset by $r_{1}=2 \mu \mathrm{m}$ from beam center. The contribution from each data set, and the in-quadrature sum of both, is much less than a fault-tolerant threshold of $10^{-4}$ for all averaging times. For laser-driven hyperfine qubits, the projected infidelities would be four times greater than the data sets presented here.

In the case where there is a small mismatch and $\Omega_{1} \neq$ $\Omega_{2}$, one must consider the effective Rabi frequency for the interaction $\widetilde{\Omega}$, where $\widetilde{\Omega} \propto \Omega_{1} \Omega_{2}[37,38]$. For a small difference in ion illumination $2 \delta \Omega_{12}$, such that $\Omega_{1}=\Omega_{0}+$ $\delta \Omega_{12}$ and $\Omega_{2}=\Omega_{0}-\delta \Omega_{12}$, then $\widetilde{\Omega} \propto\left(\Omega_{0}^{2}-\delta \Omega_{12}^{2}\right)$, where $\Omega_{0}$ is the optimum value for $\Omega_{1}$ and $\Omega_{2}$ to create the maximally-entangled state. Thus the associated error in the rotation angle on the Bloch sphere is proportional to $\delta \Omega_{12}^{2}$. In turn, it can be shown that the contribution to the Mølmer-Sørensen infidelity, due to $\delta \Omega_{12}$ alone, is [40]

$$
\left(1-F_{M S}\right)_{\text {imbalance }} \approx(\pi / 4)^{2}\left(\delta \Omega_{12} / \Omega_{0}\right)^{4}
$$

To estimate the magnitude of $\left(1-F_{M S}\right)_{\text {imbalance, }}$, we assume an inter-ion separation of $4 \mu \mathrm{m}$, with the centre of the pair offset by $r_{1}=2 \mu \mathrm{m}$ in a beam of $2 w_{0}=45 \mu \mathrm{m}$.
The largest imbalance will occur when the ion string axis is aligned radially in the beam. In such a scenario, $2 \delta \Omega_{12} / \Omega_{0}=3 \%$, which results in $\left(1-F_{M S}\right)_{\text {imbalance }}=3 \times$ $10^{-8}$, which is a similar magnitude to the infidelity contribution arising from beam position instability. By way of comparison, earlier work with ions in thermal states reported numerical simulations which predicted that an imbalance of $4 \%$ would contribute an infidelity of $\leq 10^{-4}$ [39]. These estimates suggest that our stabilisation system would reduce intensity-induced contributions to infidelity in the Mølmer-Sørensen gate to well below the level of $10^{-4}$. 


\section{Summary \& conclusions}

We have demonstrated an optical system for stabilising the intensity of a laser beam used in a pulsed-probe configuration for the coherent control of trapped ions. The system operates via two independent feedback loops; one for optical power and the other for laser beam position. Due to a cascaded arrangement of three avalanche photodiodes, the power at the ion can be measured indirectly over the range $300 \mathrm{nW} \leq P \leq 5.2 \mathrm{~mW}$, with a signalto-noise ratio $>100$. The system response is calibrated over this power range, which permits accurate setting of optical power at the ion for each pulse in a sequence. The exact position of the beam waist at the ion was stabilised by means of a quadrant photodiode and an actuated mirror. System performance was quantified by out-of-loop measurements of both power and beam position. Instability of optical power over the full range had a 2-sample fractional Allan deviation of $\sigma_{P}<2.2 \times 10^{-4}$, for averaging times $1 \mathrm{~s} \leq \tau \leq 16384 \mathrm{~s}$. In the case of the beam position, the Allan deviation yielded an absolute value of $\sigma_{r}<0.1 \mu \mathrm{m}$ (for a beam with $2 w_{0}=45 \mu \mathrm{m}$ ), for similar averaging times. This system was developed for the $674 \mathrm{~nm}$ optical qubit transition in ${ }^{88} \mathrm{Sr}^{+}$, however it is applicable to analogous species such as ${ }^{40} \mathrm{Ca}^{+}$, as well as to laser-driven hyperfine qubits in, e.g., ${ }^{9} \mathrm{Be}^{+},{ }^{43} \mathrm{Ca}^{+}$ and ${ }^{171} \mathrm{Yb}^{+}$.

There is a small risk in projecting the measured outof-loop performance to the Rabi frequency experienced by ions. While it seems reasonable to assume that the measured out-of-loop power and position fluctuations should cause commensurate intensity fluctuations at the ion position, this is not necessarily so. It is essential to guard against drifts in beamsplitter reflectivity, as well as drifts and vibrations of the ion trap with respect to the surrounding optical system. We investigated the sources likely to contribute to such errors arising from beam power; a suitably robust opto-mechanical system will prevent errors in beam pointing arising from vibrations. Intensity instability measurements using the ion response would suffer on a short term from the quantum projection noise limit, and on a longer term by other systematic drifts changing the ion response. Thus the out-of-loop measurement approach to test the long-term intensity instability is the most efficient way to establish an upper limit for that parameter.

The implications of these measurements on infidelities in quantum gate operations have been estimated by considering a Pauli $\mathrm{X}$ single qubit rotation and a twoqubit Mølmer-Sørensen gate. The minimum fidelity of the former and the fidelity of creating a 2-ion maximally entangled state with the latter have the same sensitivity to Rabi frequency instability. Concentrating on the optical qubit transitions, we calculated that the instabilities in power and beam position are expected to yield contributions to infidelities of $<3 \times 10^{-8}$ and $<3 \times 10^{-7}$ respectively, well below the generally accepted threshold of $10^{-4}$ for fault-tolerant quantum information process- 
ing. In the case of the Mølmer-Sørensen gate, a small $(\sim 3 \%)$ imbalance in the Rabi frequencies experienced by each ion is expected to contribute to the infidelity at a similar level to that of the beam position instability. Two-qubit gates based on laser-driven hyperfine transitions require a pair of laser beams interfering at the position of the trapped ions to produce a travelling standing wave parallel to the linear trap axis. Since the beams are spatially separated, each will require independent stabilisation; an independent, parallel system of APDs, quadrant photodiodes, electronics and feedback actuators will be necessary for each beam. Furthermore, the effective Rabi frequency for the stimulated Raman transition scales as the product of the pair of individual Rabi frequencies. While the analysis above has been performed for laser-driven optical qubit transitions, it is straightforward to show that hyperfine transitions driven by a stimulated Raman transition are four times more sensitive in equations $4,7,8$, and 9 . Taking this into consideration, the instability achieved here would result in an infidelity contribution of $<1.2 \times 10^{-6}$ in a hyperfine two-qubit gate.

Standard commercial laser systems have typical power instabilities no better than $1 \%$, which is insufficient for achieving an overall gate infidelity of $\leq 10^{-4}$, so a stabilisation scheme is essential. The power and position instabilities reported here may seem negligible or indeed excessively small for present applications. However, reducing all sources of infidelity well below a threshold of
$10^{-4}$ is an important goal, because the magnitude of resources required for error correction will depend on the total infidelity [2]. In the long term, it is advantageous to achieve infidelities much lower than the $10^{-4}$ threshold criterion, and in doing so minimise the overhead required for error correction.

The present system has a minimum pulse duration of $10 \mu \mathrm{s}$, which is sufficient for the duration of the highest fidelity gates $(30-100 \mu \mathrm{s})[4,11]$. Shorter pulses for fast gates [41] will require a sampling rate and a low-pass filter frequency greater than those in our apparatus. An alternative photodiode may be required to optimise signalto-noise; however, equation 8 and Fig. 8 show that $\sigma_{P}$ could increase by a factor of $\sim 10$ without a significant compromise to the overall infidelity (either $\left(1-F_{x, \text { min }}\right)$ or $\left.\left(1-F_{M S}\right)\right)$. Application to picosecond laser pulses for ultrafast qubit control [42] is beyond the present system.

Finally, the level of intensity instability demonstrated here was achieved over extended periods of duration. This is essential for the most complex investigations conducted now and in the future, and points towards a continuously operating laser system which is accurate and precise in intensity. The system presented here will be used together with a frequency stabilisation system based on a low-drift, high-finesse cavity (Stable Laser Systems) for coherent control of ${ }^{88} \mathrm{Sr}^{+}$in a microfabricated trap of similar concept to our earlier work $[35,36$, 43]. 


\section{Acknowledgements}

We thank E. Theocharous (NPL) for assistance in detector characterisation. We thank the following individuals for informative discussions: D. Szwer (NPL), D. Lucas and C. Ballance (Oxford), and C. Roos (Innsbruck). This work was funded by the UK National Measurement Office and project EXL01 QESOCAS of the European Metrology Research Programme (EMRP) [Grant Agreement No. 912/2009/EC]. The EMRP is jointly funded by the EMRP participating countries within EURAMET and the European Union. JT was supported by the EPSRC Industrial Doctorate Centre in Optics and Photonics. BY was supported by an EPSRC Knowledge Transfer Secondment from Imperial College London.

\section{References}

1. A. M. Steane, "Overhead and noise threshold of faulttolerant quantum error correction," Phys. Rev. A 68, p. 042322 , Oct 2003.

2. E. Knill, "Quantum computing with realistically noisy devices," Nature 434(7029), pp. 39-44, 2005.

3. R. Raussendorf, J. Harrington, and K. Goyal, "Topological fault-tolerance in cluster state quantum computation," New Journal of Physics 9(6), p. 199, 2007.

4. J. P. Gaebler, T. R. Tan, Y. Lin, Y. Wan, R. Bowler, A. C. Keith, S. Glancy, K. Coakley, E. Knill, D. Leibfried, and D. J. Wineland, "High-fidelity universal gate set for ${ }^{9} \mathrm{Be}^{+}$ion qubits," Phys. Rev. Lett. 117, p. 060505, Aug 2016.
5. R. Blatt and D. Wineland, "Entangled states of trapped atomic ions," Nature 453(7198), pp. 1008-1015, 2008.

6. Q. A. Turchette, C. S. Wood, B. E. King, C. J. Myatt, D. Leibfried, W. M. Itano, C. Monroe, and D. J. Wineland, "Deterministic entanglement of two trapped ions," Phys. Rev. Lett. 81(17), pp. 3631-3634, 1998.

7. C. A. Sackett, D. Kielpinski, B. E. King, C. Langer, V. Meyer, C. J. Myatt, M. Rowe, Q. A. Turchette, W. M. Itano, D. J. Wineland, and C. Monroe, "Experimental entanglement of four particles," Nature 404(6775), pp. $256-259,2000$.

8. M. A. Rowe, D. Kielpinski, V. Meyer, C. A. Sackett, W. M. Itano, C. Monroe, and D. J. Wineland, "Experimental violation of a Bell's inequality with efficient detection," Nature 409(6822), pp. 791-794, 2001.

9. D. Leibfried, B. DeMarco, V. Meyer, D. Lucas, M. Barrett, J. Britton, W. M. Itano, B. Jelenkovic, C. Langer, R. T., and D. J. Wineland, "Experimental demonstration of a robust, high-fidelity geometric two ion-qubit phase gate," Nature 422(6930), pp. 412-415, 2003.

10. J. Benhelm, G. Kirchmair, C. F. Roos, and R. Blatt, "Towards fault-tolerant quantum computing with trapped ions," Nature Phys. 4, pp. 463-466, 2008.

11. C. J. Ballance, T. P. Harty, N. M. Linke, M. A. Sepiol, and D. M. Lucas, "High-fidelity quantum logic gates using trapped-ion hyperfine qubits," Phys. Rev. Lett. 117, p. 060504, Aug 2016. Also see Supplemental Material at http://link.aps.org/supplemental/10.1103/PhysRevLett.117.060504

12. E. Knill, "Physics: quantum computing," Nature 463(7280), pp. 441-443, 2010.

13. T. D. Ladd, F. Jelezko, R. Laflamme, Y. Nakamura, C. Monroe, and J. L. O'Brien, "Quantum computers," 
Nature 464, pp. 45-53, 2010.

14. D. Leibfried, R. Blatt, C. Monroe, and D. Wineland, "Quantum dynamics of single trapped ions," Rev. Mod. Phys. 75(1), pp. 281-324, 2003.

15. K. R. Brown, A. C. Wilson, Y. Colombe, C. Ospelkaus, A. M. Meier, E. Knill, D. Leibfried, and D. J. Wineland, "Single-qubit-gate error below $10^{-4}$ in a trapped ion," Phys. Rev. A 84, p. 030303, Sep 2011.

16. T. P. Harty, D. T. C. Allcock, C. J. Ballance, L. Guidoni, H. A. Janacek, N. M. Linke, D. N. Stacey, and D. M. Lucas, "High-fidelity preparation, gates, memory, and readout of a trapped-ion quantum bit," Phys. Rev. Lett. 113, p. 220501, Nov 2014.

17. T. R. Tan, J. P. Gaebler, Y. Lin, Y. Wan, R. Bowler, D. Leibfried, and D. J. Wineland, "Multi-element logic gates for trapped-ion qubits," Nature 528(7582), pp. 380-383, 2015.

18. T. R. Tan, J. P. Gaebler, R. Bowler, Y. Lin, J. D. Jost, D. Leibfried, and D. J. Wineland, "Demonstration of a dressed-state phase gate for trapped ions," Phys. Rev. Lett. 110, p. 263002, Jun 2013.

19. A. C. Wilson, Y. Colombe, K. R. Brown, E. Knill, D. Leibfried, and D. J. Wineland, "Tunable spin-spin interactions and entanglement of ions in separate potential wells," Nature 512(7512), pp. 57-60, 2014.

20. P. Schindler, D. Nigg, T. Monz, J. T. Barreiro, E. Martinez, S. X. Wang, S. Quint, M. F. Brandl, V. Nebendahl, C. F. Roos, et al., "A quantum information processor with trapped ions," New J. Phys. 15(12), p. 123012, 2013.

21. N. Akerman, N. Navon, S. Kotler, Y. Glickman, and R. Ozeri, "Universal gate-set for trapped-ion qubits us- ing a narrow linewidth diode laser," New J. Phys. 17(11), p. $113060,2015$.

22. Z. Meir, O. Schwartz, E. Shahmoon, D. Oron, and R. Ozeri, "Cooperative Lamb shift in a mesoscopic atomic array," Phys. Rev. Lett. 113, p. 193002, Nov 2014.

23. J. Thom, G. Wilpers, E. Riis, and A. G. Sinclair, "Accurate and agile digital control of optical phase, amplitude and frequency for coherent atomic manipulation of atomic systems," Opt. Express 21, pp. 18712-18723, Aug 2013.

24. E. Theocharous and E. M. Wareham, "Ultra-high performance photodetection systems for radiometric applications," Meas. Sci. Technol. 15(6), p. 1216, 2004.

25. E. Theocharous, J. Ishii, and N. P. Fox, "Absolute linearity measurements on $\mathrm{HgCdTe}$ detectors in the infrared region," Appl. Opt. 43, pp. 4182-4188, Jul 2004.

26. E. Theocharous, "Absolute linearity measurements on a PbS detector in the infrared," Appl. Opt. 45, pp. 23812386, Apr 2006.

27. E. Theocharous, M. A. Itzler, J. Cheung, and C. J. Chunnilall, "Characterization of the linearity of response and spatial uniformity of response of two InGaAsP/InP Geiger-mode avalanche photodiodes," IEEE J. Quant. Electron. 46, pp. 1561-1567, Nov 2010.

28. C. F. Roos, "Ion trap quantum gates with amplitudemodulated laser beams," New J. Phys. 10(1), p. 013002, 2008.

29. D. B. Leviton and B. J. Frey, "Temperature-dependent absolute refractive index measurements of synthetic fused silica," Proc. SPIE 6273, pp. 62732K-62732K-11, 2006. 
30. B. J. Frey, D. B. Leviton, T. J. Madison, Q. Gong, and M. Tecza, "Cryogenic temperature-dependent refractive index measurements of N-BK7, BaLKN3, SF15, and ESF03," Proc. SPIE 6692, pp. 669205-669205-12, 2007.

31. D. Allan, "Statistics of atomic frequency standards," Proc. of the IEEE 54, pp. 221-230, Feb 1966.

32. TEM Messtechnik GmbH http://www.temmesstechnik.de/EN/aligna.htm.

33. R. Walder, D. H. Paik, M. S. Bull, C. Sauer, and T. T. Perkins, "Ultrastable measurement platform: subnm drift over hours in $3 \mathrm{~d}$ at room temperature," Opt. Express 23, pp. 16554-16564, Jun 2015.

34. E. Mount, C. Kabytayev, S. Crain, R. Harper, S.-Y. Baek, G. Vrijsen, S. T. Flammia, K. R. Brown, P. Maunz, and J. Kim, "Error compensation of single-qubit gates in a surface-electrode ion trap using composite pulses," Phys. Rev. A 92, p. 060301, Dec 2015.

35. G. Wilpers, P. See, P. Gill, and A. G. Sinclair, "A monolithic array of three-dimensional ion traps fabricated with conventional semiconductor technology," Nature Nanotech. 7, pp. 572-576, 2012.

36. G. Wilpers, P. See, P. Gill, and A. G. Sinclair, "A compact UHV package for microfabricated ion-trap arrays with direct electronic air-side access," Appl. Phys. B 111(1), pp. 21-28, 2013.

37. K. Mølmer and A. Sørensen, "Multiparticle entanglement of hot trapped ions," Phys. Rev. Lett. 82, pp. 18351838, Mar 1999.

38. A. Sørensen and K. Mølmer, "Entanglement and quantum computation with ions in thermal motion," Phys. Rev. A 62, p. 022311, Jul 2000.
39. G. Kirchmair, J. Benhelm, F. Zhringer, R. Gerritsma, C. F. Roos, and R. Blatt, "Deterministic entanglement of ions in thermal states of motion," New Journal of Physics 11(2), p. 023002, 2009.

40. C. F. Roos, private communication.

41. M. Palmero, S. Martínez-Garaot, D. Leibfried, D. J. Wineland, and J. G. Muga, "Fast phase gates with trapped ions," Phys. Rev. A 95, p. 022328, Feb 2017.

42. J. Mizrahi, B. Neyenhuis, K. G. Johnson, W. C. Campbell, C. Senko, D. Hayes, and C. Monroe, "Quantum control of qubits and atomic motion using ultrafast laser pulses," Applied Physics B 114(1), pp. 45-61, 2014.

43. P. See, G. Wilpers, P. Gill, and A. G. Sinclair, "Fabrication of a monolithic array of three dimensional Si-based ion traps," J. Microelectromech. Syst. 22, pp. 1180-1189, 2013. 